\title{
Metodologias para a criação de jogos educativos: uma revisão sistemática da literatura
}

\author{
Samara de Sena \\ Programa de Pós-Graduação em Engenharia e Gestão do conhecimento, Universidade Federal de Santa \\ Catarina, senasami@gmail.com \\ Araci Hack Catapan \\ Programa de Pós-Graduação em Engenharia e Gestão do conhecimento, Universidade Federal de Santa \\ Catarina, aracihack@gmail.com
}

Resumo: Esta pesquisa propõe, por meio da técnica de revisão sistemática da literatura, um mapeamento sobre metodologias para o desenvolvimento de jogos educativos, enfocando, principalmente, em estudos que explicitam a concepção da documentação de design. Como resultado foram recuperados dez estudos que foram divididos em dois eixos temáticos: (1) estudos que propõem metodologias para a criação de jogos educativos; e (2) estudos sobre o desenvolvimento de jogos educativos que descrevem a metodologia utilizada no processo de design. Concluiu-se que não existe um padrão metodológico para o design desse tipo de artefato e que muitas metodologias consolidadas em outras áreas do conhecimento são adaptadas com eficiência no design de jogos para a educação.

Palavras-chave: jogos educativos, design de jogos, metodologias.

\section{Methodologies to the creation of educational games: a systematic review of the literature}

Abstract: This research proposes, through systematic review of literature, a mapping on methodologies for the development of educational games, focusing mainly on studies that explain the conception of the design documentation. As a result, it was possible to recover ten studies that were divided into two themes: (1) studies proposing methodologies for creating educational games; and (2) research on the development of an educational game that describe the method used in the design process. It was concluded that there isn't a methodological pattern for that type of artifact design, and many consolidated methodologies in other areas of knowledge are adapted effectively to the development of educational games.

Keywords: educational games, game design, methodologies.

\section{INTRODUÇão}

A utilização de jogos digitais é uma atividade consolidada em diversos contextos educacionais. Os últimos relatórios Horizon Report apontam o uso de games, gamificação e aprendizagem baseada em jogos digitais como tendências para a educação a partir de 2012 no ensino fundamental e médio das escolas brasileiras (Johnson et al., 2012); a partir de 2014 nas escolas da Europa (Johnson et al., 2014); e a partir de 2015 nas escolas da Ásia (Becker et al., 2016) e no ensino superior brasileiro (Freeman et al., 2015).

$\mathrm{Na}$ direção dessa tendência, o design de jogos educativos se tornou uma atividade de pesquisa recorrente nas universidades, englobando as mais diversas áreas do conhecimento. No entanto, pela sua natureza, essa prática demanda o estudo e a elaboração de metodologias próprias para o desenvolvimento desses artefatos. 
Independentemente de ser objeto de pesquisa ou produto de entretenimento, o design de jogos é uma atividade inerentemente interdisciplinar, abrangendo conhecimentos e profissionais de diversas áreas, trabalhando cooperativamente. Para que esse processo e, sobretudo, a comunicação entre esses profissionais seja efetiva, é necessário que se observe alguns critérios metodológicos, entre eles, a documentação, como o documento de conceito, o documento técnico, o guia de estilo de arte e o game design document (GDD) (Novak, 2008). A quantidade, bem como, o nível de complexidade desses documentos variam de acordo com o perfil e tamanho de projeto. No entanto, o GDD é o documento mais importante a ser produzido, pois contém todas as informações necessárias para que a equipe de desenvolvimento consiga desempenhar suas tarefas e, ao mesmo tempo, mantenha uma visão abrangente sobre o artefato em processo.

Esta pesquisa objetiva, por meio da técnica de revisão sistemática da literatura, elencar metodologias utilizadas no desenvolvimento de jogos para a educação, buscando, sobretudo, mapear estudos que relatam o processo de documentação de design.

\section{Metodologia}

Este estudo foi desenvolvido pelo método de revisão sistemática da literatura. Essa metodologia objetiva o mapeamento, avaliação crítica e a agregação de estudos relevantes realizados anteriormente, sobre o tema em estudo. O propósito é verificar o avanço conceitual ou prático do tema e identificar lacunas. Os resultados podem ser expressos como uma síntese sobre a questão e também ser um indicador para novos estudos. Nesse sentido, a metodologia tem como premissa o uso de um método planejado e explícito, para evitar que haja viés nos resultados, e tem como benefício a geração de uma visão abrangente sobre o tema estudado e possíveis estudos em sequência (Morandi; Camargo, 2015).

Encontra-se na literatura diversos modos de realizar uma revisão sistemática. Esse estudo foi realizado com base nos passos propostos por Costa e Zoltowski (2015), conforme mostra a figura 01 .

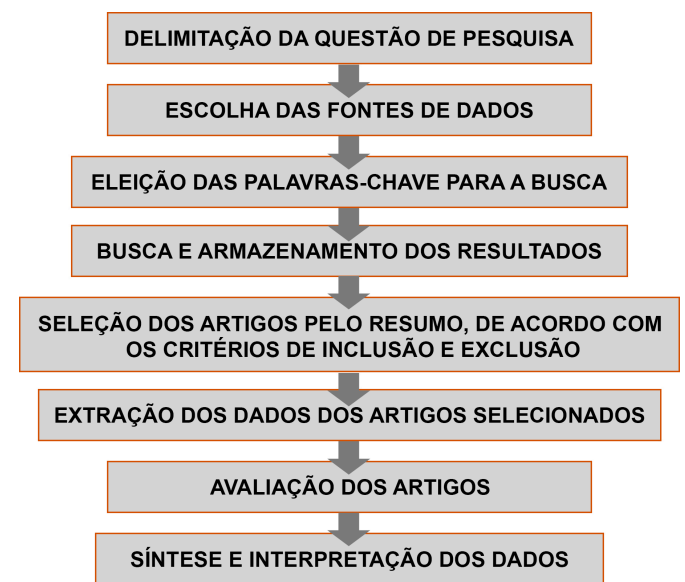

Figura 1- Etapas da revisão sistemática com base em Costa e Zoltowski (2015).

Neste caso essa revisão sistemática da literatura objetiva mapear estudos que descrevem metodologias utilizadas no design de jogos educacionais, em diversas profundidades, buscando, sobretudo, identificar trabalhos que descrevem a elaboração da documentação de design feita para esse tipo de jogos. Para isso elaborou-se a 
seguinte questão de pesquisa: que metodologias são empregadas para a criação de jogos educacionais?

Nesse estudo, optou-se pela manutenção do termo game design document, por ser um termo técnico adotado sem tradução para a língua portuguesa.

\subsection{ESTRATÉGIAS DE BUSCA}

Devido à quantidade de informações a serem gerenciadas em uma revisão sistemática, Morandi e Camargo (2015) orientam que, no processo de pesquisa, é necessário partir de uma estratégia de busca previamente estabelecida.

Para essa pesquisa foram escolhidas as seguintes bases científicas: Scielo, IEEE, Web of Science e ERIC, devido a relevância dessas bases nas áreas de educação, tecnologia e interdisciplinar. As buscas foram realizadas em agosto de 2016, a partir das três estratégias explicitadas no quadro 1.

\begin{tabular}{|c|c|c|}
\hline & & Quadro 1- Estratégias de busca \\
\hline Busca & Bases & Estratégia \\
\hline \multirow[t]{2}{*}{$\begin{array}{l}\text { Primeira } \\
\text { Estratégia }\end{array}$} & $\begin{array}{l}\text { Bases: IEEE, Web } \\
\text { of Science e ERIC }\end{array}$ & $\begin{array}{l}\text { "game design document" OR GDD AND "serious games" OR } \\
\text { "educational games" }\end{array}$ \\
\hline & Base: Scielo & $\begin{array}{l}\text { "game design document" OR GDD AND "jog* educa*" OR } \\
\text { "jogos sérios" OR "aprendizagem baseada em jogos digitais" }\end{array}$ \\
\hline \multirow[t]{2}{*}{$\begin{array}{l}\text { Segunda } \\
\text { estratégia }\end{array}$} & $\begin{array}{l}\text { Bases: IEEE, Web } \\
\text { of Science e ERIC }\end{array}$ & documentation AND “serious games” OR “educational games” \\
\hline & Base: Scielo & $\begin{array}{l}\text { "game design document" OR GDD AND "jog* educa*" OR } \\
\text { "jog* séri*" OR "aprendizagem baseada em jogos digitais" }\end{array}$ \\
\hline \multirow[t]{2}{*}{$\begin{array}{l}\text { Terceira } \\
\text { estratégia }\end{array}$} & $\begin{array}{l}\text { Bases: IEEE, Web } \\
\text { of Science e ERIC }\end{array}$ & game design AND methodology AND “educational games” \\
\hline & Base: Scielo & $\begin{array}{l}\text { "game design" AND “jog* séri*” OR “jog* digita*” OR "gam* } \\
\text { educa*” OR "jog* educa*" }\end{array}$ \\
\hline
\end{tabular}

Para a seleção, foram considerados os seguintes critérios de inclusão: (1) somente artigos; (2) escritos em inglês, português ou espanhol; (3) publicados entre os anos de 2012 e 2016; (5) que disponibilizassem o texto completo, de forma gratuita; e (6) que fossem revisados por pares (opção da base ERIC).

\section{RESUltados}

Como resultado das buscas, as bases retornaram 252 artigos dos quais, a partir da aplicação dos critérios de inclusão e da remoção de trabalhos duplicados, foram recuperados 10 estudos (fig. 2). 


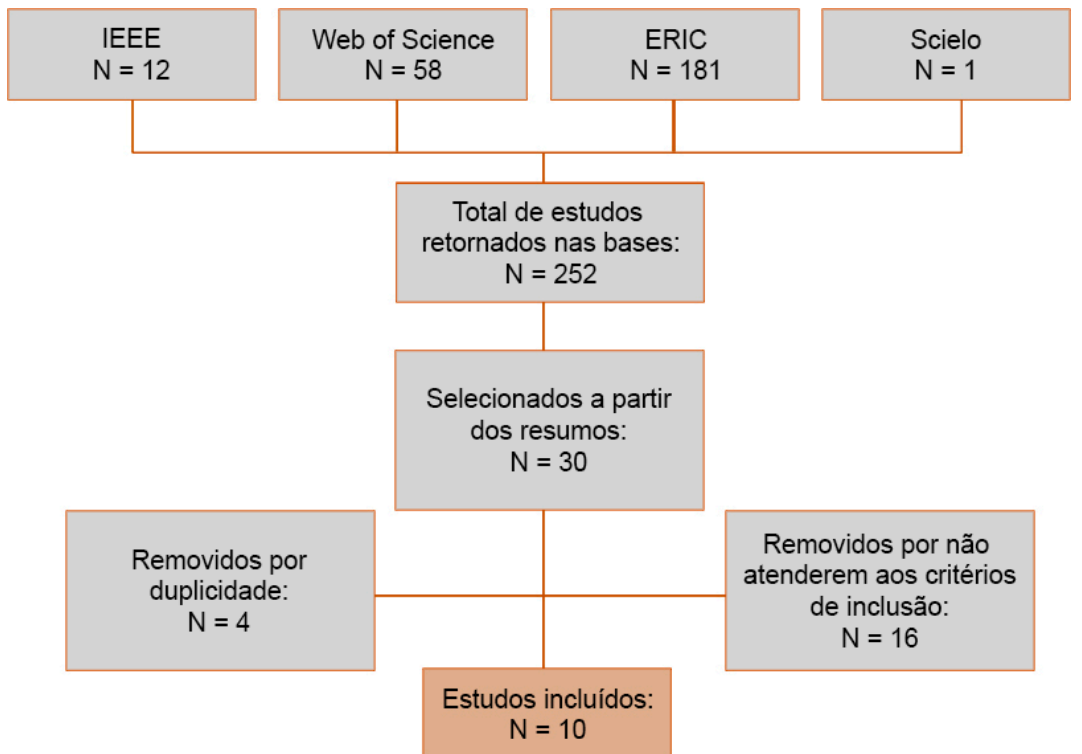

Figura 2 - Resultados da revisão sistemática

Os estudos incluídos passaram por uma segunda leitura analítica, dessa vez com base nos textos completos. Após essa análise, foram agrupados e codificados para a elaboração da síntese dos resultados (quadro 2).

Foram identificados 02 eixos temáticos: (1) estudos que propõe metodologias para a criação de jogos educacionais; e (2) estudos sobre o desenvolvimento de algum jogo educacional que descreve, em qualquer nível de profundidade, a metodologia utilizada no processo de design.

Quadro 2 - Estudos incluídos por eixo temático

\begin{tabular}{|c|c|c|}
\hline Base & Títulos e link para o artigo & Eixo temático \\
\hline $\begin{array}{l}\text { Web of } \\
\text { Science }\end{array}$ & $\begin{array}{l}\text { Cognitive behavioral game design: a unified model for designing } \\
\text { serious games. Disponível em: < } \\
\text { https://www.ncbi.nlm.nih.gov/pmc/articles/PMC3910127>. }\end{array}$ & $\begin{array}{l}\text { Proposição } \\
\text { metodologias }\end{array}$ \\
\hline IEEE & $\begin{array}{l}\text { Development of game-like simulations for procedural knowledge in } \\
\text { healthcare education. Disponível em: } \\
<\text { http://ieeexplore.ieee.org/document } / 6678341 />\text {. }\end{array}$ & $\begin{array}{l}\text { Proposição } \\
\text { metodologias }\end{array}$ \\
\hline $\begin{array}{l}\text { Web of } \\
\text { Science }\end{array}$ & 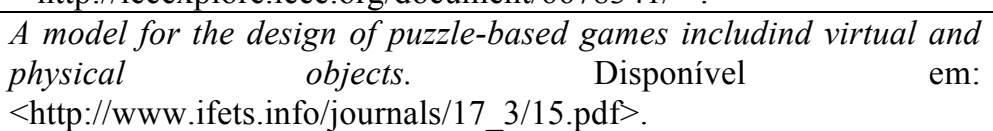 & $\begin{array}{l}\text { Proposição } \\
\text { metodologias }\end{array}$ \\
\hline ERIC & $\begin{array}{l}\text { A plataform independent game technology model for model driven } \\
\text { serious games development. Disponível em: }< \\
\text { www.ejel.org/issue/download.html?idArticle=228>. }\end{array}$ & $\begin{array}{l}\text { Proposição } \\
\text { metodologias }\end{array}$ \\
\hline $\begin{array}{l}\text { Web of } \\
\text { Science }\end{array}$ & $\begin{array}{l}\text { Using serious game to enhance algorithmic learning and teaching. } \\
\text { Disponível em: < http://www.je-lks.org/ojs/index.php/Je- } \\
\text { LKS_EN/article/view/1125>. }\end{array}$ & $\begin{array}{l}\text { Relato de design de } \\
\text { um jogo com } \\
\text { descrição } \\
\text { metodológica }\end{array}$ \\
\hline $\begin{array}{l}\text { Web of } \\
\text { Science }\end{array}$ & 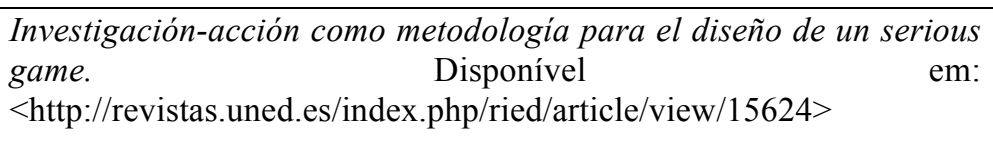 & $\begin{array}{l}\text { Relato de design de } \\
\text { um jogo com } \\
\text { descrição } \\
\text { metodológica }\end{array}$ \\
\hline ERIC & $\begin{array}{l}\text { Development of a computer simulation game using a reverse } \\
\text { engineering approach. Disponível em: } \\
<\text { http://www.cluteinstitute.com/ojs/index.php/AJBE/article/view/738 } \\
7>\text {. }\end{array}$ & $\begin{array}{l}\text { Relato de design de } \\
\text { um jogo com } \\
\text { descrição } \\
\text { metodológica }\end{array}$ \\
\hline ERIC & $\begin{array}{l}\text { A digital game-based learning system for energy education: an } \\
\text { energy conservation pet. } \\
\text { Disponível em: }<\text { http://www.tojet.net/articles/v11i2/1123.pdf }>\text {. }\end{array}$ & $\begin{array}{l}\text { Relato de design de } \\
\text { um jogo com } \\
\text { descrição } \\
\text { metodológica }\end{array}$ \\
\hline
\end{tabular}




\begin{tabular}{|l|l|l|}
\hline ERIC & $\begin{array}{l}\text { Employing design and development research (DDR) approaches in } \\
\text { the design and development of online Arabic vocabulary learning } \\
\text { games } \\
\text { http://www.tojet.net/articles/v11i2/11211.pdf }>\text {. }\end{array}$ & $\begin{array}{l}\text { Relato de design de } \\
\text { um jogo com } \\
\text { descrição } \\
\text { metodológica }\end{array}$ \\
\hline ERIC & $\begin{array}{l}\text { Implementing a game for supporting learning in mathematics. } \\
\text { Disponível em: } \\
\text { <www.ejel.org/issue/download.html?idArticle=284 }>.\end{array}$ & $\begin{array}{l}\text { Relato de design de } \\
\text { um jogo com } \\
\text { descrição } \\
\text { metodológica }\end{array}$ \\
\hline
\end{tabular}

\section{SÍNTESE DOS RESULTADOS}

Para a apresentação dos resultados foi realizada uma síntese textual narrativa. Nessa abordagem, os estudos são agrupados a partir de um relato estruturado dos padrões identificados (Morandi; Camargo, 2015).

\subsection{ESTUDOS QUE PROPÕE METODOLOGIAS PARA A CRIAÇÃO DE JOGOS EDUCACIONAIS}

Neste eixo foram identificados quatro trabalhos que resultaram de pesquisas sobre a proposição de diferentes metodologias, frameworks e modelos para a criação de jogos educacionais.

Melero e Hernandéz-Leo (2014), propõe um modelo para o design de jogos baseados em puzzles, que são jogos com regras fechadas e mecânicas simples, como os quebra-cabeças. O modelo proposto pelos autores prevê a utilização de objetos físicos ou virtuais e é direcionado para professores que queiram criar seus próprios jogos. Segundo os autores, jogos educacionais que estão disponíveis não abarcam a quantidade e complexidade de temas que os professores precisam abordar em suas aulas, então, através de ferramentas que facilitam o design de jogos autorais, a criação de jogos pelos próprios professores pode ser tornar uma opção viável (Melero; Hernández-Leo, 2014).

Os autores propõe seu modelo com base em puzzles, pois consideram que são jogos com mecânicas fáceis, tanto do ponto de vista de design, quanto de entendimento pelo usuário. No artigo, os autores descrevem, através de um framework, a visão geral sobre o modelo proposto e ainda fornecem um documento XMA para a sua representação computacional, que detalha o modelo conceitual para que os professores consigam fazer a aplicação em ferramentas autorais para jogos digitais. Esta parte da metodologia, porém, requer algum nível de conhecimento de ferramentas computacionais que não está ao alcance de todos os professores, podendo ser necessária a ajuda de um especialista. No artigo, os autores também mostram diversas aplicações do modelo, tanto em jogos virtuais como físicos, exemplificando como a aprendizagem baseada em puzzles pode ser diversificada.

Este trabalho exemplifica como a aprendizagem por jogo não precisa se limitar à mecânicas complexas, que demandam equipes e meses de desenvolvimento. Mecânicas simples, como as dos puzzles, podem ser recursos valiosos de ensino-aprendizagem, se planejadas de forma que sejam interessantes para os usuários.

Tang et al. (2013) também apresentam um modelo para o desenvolvimento de jogos educativos, porém, com foco na área de desenvolvimento de software. Os autores propõe um modelo que permite a interoperabilidade entre soluções de software, independente da plataforma de hardware ou especificações operacionais, para a criação de jogos educativos. O framework de desenvolvimento de jogos proposto no estudo que consiste em nove partes: (1) interface, (2) modelos, (3) ferramentas, (4) bibliotecas de componentes, (5) padrões de códigos, (6) artefatos, (7) plataformas de tecnologia, (8) plataforma de operação e (9) software (Tang et al, 2013). Porém, como o modelo 
proposto pelos autores têm foco exclusivo na área de tecnologia, não se aprofunda nas questões relativas ao design.

$\mathrm{Na}$ área de psicologia, Starks (2014) propõe o modelo cognitive behavioral game design (CBGD). Nele, a autora propõe um framework para a criação de jogos sérios que inclui uma combinação entre 5 elementos da teoria social cognitiva, 11 elementos da teoria das múltiplas inteligências de Gardner e 5 princípios de design de jogos.

O artigo de Starks (2014) aprofunda cada elemento do modelo CBGD e como estes podem ser aplicados no design de jogos educativos. Além disso, oferece exemplos de aplicação, provendo uma avaliação do uso modelo. O estudo corrobora, ainda, com a importância da associação entre conceitos de aprendizagem e de diversão para o sucesso dos jogos para a educação.

Torrente et al. (2014) apresentam a metodologia educational game development approach (EGDA), para a criação de jogos educativos para a aprendizagem de conhecimentos processuais na área de saúde. "Conhecimento processual pode ser definido como o conhecimento que é aplicado no desenvolvimento de um procedimento ou uma sequência de ações para atingir um objetivo" (Torrent et al., 2014).

A metodologia EGDA cobre todas as etapas de produção de um jogo, desde as tarefas de game design, até a implementação e avaliação, e está baseada em quatro princípios básicos: (1) aproximação centrada no procedimento, (2) colaboração entre especialistas, (3) desenvolvimento ágil e ferramentas de autoria e (4) modelo de jogo de baixo custo (Torrent et al., 2014). Além das descrições sobre os quatro princípios básicos, os autores discorrem detalhadamente sobre cada fase envolvida no processo de implementação da metodologia.

Este artigo apresentou uma metodologia detalhada para a elaboração de jogos que, apesar de ter sido elaborada tendo em vista a área de saúde, pode ser aplicada a diversas outras áreas do conhecimento que precisam focar na aprendizagem de procedimentos precisos. O trabalho também se destaca por se preocupar em propor um sistema que minimiza o custo de desenvolvimento, sendo bastante interessante para a utilização em contextos acadêmicos que nem sempre contam com os mesmos recursos financeiros e humanos que as empresas de desenvolvimento podem arcar.

\subsection{ESTUDOS QUE DESCREVEM O DESIGN DE JOGOS COM EXPLICITAÇÃO DA METODOLOGIA UTILIZADA}

Neste eixo temático foram incluídos seis trabalhos que relatam o processo de design de jogos educativos destinados a diversas áreas do conhecimento e que, em qualquer nível de profundidade, relatam as etapas e/ou métodos empregados no processo de criação dos artefatos.

Debabi e Bensebaa (2016) descrevem o processo de criação do jogo AlgoGame. O jogo é destinado aos estudantes que estão iniciando na área de programação. Os autores relatam que o aprendizado de algoritmos e os fundamentos da área de ciência da computação são difíceis de ensinar e aprender e que muitos estudantes dos programas de computação apresentam dificuldade de aprendizagem e falta de motivação, especialmente no começo do curso. Sendo assim, os autores, partindo do pressuposto de que os jogos possuem o poder de engajar e motivar, propuseram o jogo AlgoGame, para os estudante que estão tendo seu primeiro contato com as noções de programação. $\mathrm{O}$ jogo objetiva introduzir conceitos de algoritmo e ajudar os estudantes a entender a forma como os algoritmos são estruturados (Debabi; Bensebaa, 2016).

Em seu artigo, os autores não chegam a descrever as etapas de desenvolvimento do jogo, mas apresentam uma descrição sobre as mecânicas elencadas para seu 
funcionamento. Percebeu-se a intenção de prover o jogador com um ambiente de jogo que remete ao seu ambiente natural, o departamento de ciência da computação, porém, o personagem e as missões dão conta dos aspectos lúdicos e desafiadores que um jogo deve proporcionar. Apesar de não haver detalhamento sobre o processo de design, a descrição da etapa de pesquisa e das mecânicas tornam o estudo apto a servir como referencial para o desenvolvimento de outros jogos similares.

Contreras-Espinosa e Eguia-Gómez (2016) descrevem o design de um jogo com base na metodologia de pesquisa-ação. Nesse processo, os pesquisadores incluíram professores do ensino fundamental no design do jogo, em um processo coparticipativo. O desenvolvimento foi realizado em dois ciclos, cada um contando com cinco etapas: (1) diagnóstico, (2) planejamento das ações, (3) ações, (4) avaliação e (5) especificações.

Como resultado, foi criado um jogo para crianças de 8 a 11 anos de idade, tendo como tema a industrialização da Cataluña. O jogo, conforme relaram os autores, contém 14 níveis e segue a estrutura de plataforma. Além dos conteúdos pedagógicos, também contribui para o desenvolvimento psicomotor, orientação espacial, senso de direção e atenção, assim como rapidez de resposta (Contreras-Espinosa; Eguia-Gómez, 2016).

Os autores apresentam um relato sobre o complexo processo de design do jogo e descreveram com detalhes suas etapas, dando ênfase aos processos de pesquisa, prototipação e documentação. Além disso, dão subsídios de como utilizar a pesquisaação como uma metodologia que permite a participação dos usuários no processo de design.

Yang et al. (2012) descrevem o design do jogo ECOPET, que foi desenvolvido com o objetivo de aumentar o conhecimento dos usuários a respeito da conservação de energia elétrica em casa. O objetivo do jogo consiste em, através do cuidado de um mascote (pet), levar os usuários a situações que desenvolvem a consciência sobre medidas de conservação de energia. $\mathrm{O}$ mascote interage com o jogador em quatro cenários de uma casa que, por sua vez, contém vários tipos de eletrodomésticos. A mecânica do jogo se baseia em cuidados do mascote que envolvem o uso racional de aparelhos elétricos. Em cada jogada, o jogo fornece cotas de uso de energia para que o usuário aprenda a administrar o uso.

Como o nível de conhecimento a respeito do uso de energia varia entre os usuários, o design do jogo foi pensado de forma a fornecer diferentes feedbacks e mecanismos de complexidade nos desafios. Os feedbacks visam fornecer dicas sobre o consumo racional de energia para cada aparelho elétrico disponibilizado no cenário. $\mathrm{O}$ design contempla diferentes níveis de complexidade de acordo com o número de feedbacks fornecidos, adequando o grau de dificuldade do jogo de acordo com o nível de conhecimento do jogador (Yang et al., 2012).

Apesar de não ter contemplado o processo de design do artefato de forma aprofundada, o artigo contribui como possível referência para o desenvolvimento de jogos similares.

Sahrir et al. (2012) relatam o processo de design e desenvolvimento de um protótipo de jogo para a aprendizagem online de vocabulário árabe. O protótipo envolve a integração de conteúdos de aprendizagem do vocabulário árabe que é encontrado em livros tradicionais, dentro da ferramenta de autoria Raptivity, que oferece uma série de templates para a criação de jogos.

Os pesquisadores se basearam na metodologia design and development research (DDR) para a criação do protótipo, por ser uma metodologia ao mesmo tempo sistemática e flexível para a melhoria de práticas educacionais (Sahrir et al, 2012). Nesse sentido, o desenvolvimento com base na metodologia DDR contou com as 
seguintes premissas: (1) desenvolvimento teórico multi e interdisciplinar; (2) modelo de métodos mistos; (3) trabalho em equipe cíclico e iterativo; (4) literatura extensiva, colaboração, parceria e pesquisa de tecnologias; e (5) melhoria da teoria e produção com base em princípios de design (Sahrir et al., 2012).

$\mathrm{O}$ estudo enfatizou que o uso de ferramentas de autoria, mesmo com algumas restrições, pode ser um recurso acessível para pesquisadores e professores que queiram desenvolver seus próprios jogos sem a dependência de especialistas em tecnologia para a criação de códigos.

Katmada et al. (2014) apresentam um relato sobre o processo de design, desenvolvimento e avaliação do jogo Volcanic Riddles ', cujo objetivo é a facilitação da aprendizagem da matemática nos ensinos fundamental e médio. Segundo pesquisas realizadas pelos autores, a aprendizagem de matemática ainda enfrenta dificuldades, sendo um problema entre estudantes de todas as idades. Além do jogo, foi desenvolvido um website de apoio aos professores que, sem uso de programação, são habilitados a customizar configurações do jogo, podendo personalizá-lo de acordo com necessidades de aprendizagem e níveis de dificuldade específicos.

O estudo se divide em duas partes: na primeira parte os autores tratam do processo de desenvolvimento de um protótipo de jogo que visa ser uma ferramenta educacional adaptável e, na segunda parte, tratam da avaliação do protótipo, feita a partir de testes com estudantes.

O modelo ADDIE foi utilizado para guiar o processo de desenvolvimento do jogo, sendo assim, de acordo com as etapas do modelo, o seguinte framework foi adotado: análise, design, desenvolvimento, implementação e avaliação. $\mathrm{Na}$ etapa de design do jogo, os autores fizeram pesquisas sobre game design, buscando criar um produto que cumprisse princípios básicos de jogos digitais, para que além de educacional, fosse também motivador para os usuários.

Foi verificada a importância do conhecimento de princípios de design de jogos por parte de pesquisadores que objetivam criar jogos voltados para a educação. Além disso, verificou-se que o uso do modelo ADDIE se adequa ao design de jogos educativos.

Outra metodologia adaptada para o desenvolvimento de jogos foi utilizada por Ozkul (2012), que se apropriou de conceitos da engenharia reversa para o desenvolvimento de um jogo de simulação sobre administração. O jogo se baseia em um módulo do jogo PROSIM III, porém, foi criado para conter menos módulos e eliminar regras muito complexas.

O conceito de engenharia reversa se baseia na extração de conhecimento e padrões de design de qualquer artefato criado pelo homem, através do processo de análise de seus componentes e suas relações. Após essa análise, é criado um novo sistema com outra forma e um nível de abstração mais elevado (Ozkul, 2012). Segundo o autor, a engenharia reversa é normalmente utilizada para produtos físicos, porém, no desenvolvimento de software, é útil para o entendimento da estrutura, operações e comportamento dos softwares analisados.

Para o desenvolvimento do jogo, foi criado um plano baseado em princípios de engenharia reversa: (1) lista de características e regras sobre a lógica do jogo interno documentado pela revisão do manual do usuário do jogo em estudo; (2) identificação de recursos não documentados, através da análise da jogabilidade; (3) discussão sobre outras características e comportamentos não recuperados pelos passos anteriores e (4) desenvolvimento e concepção de características equivalentes a essas partes do jogo. Após a identificação das especificações de design do jogo original por meio destas fases iniciais de análise, um novo jogo foi desenvolvido. O PROSYS , como foi chamado, contém as características do jogo original, com algumas melhorias, tais como os 
gráficos, a interface e a usabilidade geral (Ozkul, 2012).

$\mathrm{O}$ estudo, apesar de ter enfoque técnico na área de tecnologia, corrobora para a efetividade na adaptação de metodologias consolidadas em outras áreas, como base metodológica para a concepção de jogos. Também contribui para o estímulo à prática da observação e análise de características de jogos já conceituados, visando a apropriação e melhorias de seus recursos.

\section{CONSIDERAÇÕES FINAIS}

Este estudo buscou, por meio da técnica de revisão sistemática da literatura, elencar metodologias para a criação de jogos digitais para a educação, sobretudo os que relatam o processo de documentação de design. Como resultado, foram incluídos 10 estudos, que foram posteriormente agrupados em dois eixos temáticos.

$\mathrm{O}$ primeiro eixo temático apresentou quatro estudos que propõe novas metodologias para a criação de jogos. O destaque neste grupo de estudos se deu pela variedade de áreas do conhecimento propondo essas metodologias, o que demonstra que a disseminação da prática do design de jogos no âmbito acadêmico e a necessidade de metodologias com abordagens cada vez mais interdisciplinares.

O segundo eixo temático agrupou seis estudos que relatam a criação de jogos na educação, com explicitação da metodologia utilizada. Neste eixo teve destaque a apropriação e adaptação de metodologias já existentes e consolidadas para a produção de outros artefatos, como a metodologia ADDIE, largamente utilizada no design instrucional, ou mesmo a engenharia reversa, consolidada na área de tecnologia.

Um fator relevante nos dois eixos mapeados foi a falta de estudos sobre os processos de documentação de design, sobretudo sobre a criação do GDD desses jogos.

Esse estudo mostra que o design de jogos está cada vez mais consolidado como prática de pesquisa, abrangendo variadas metodologias e áreas do conhecimento. Outra conclusão é que o design de jogos para a educação não precisa seguir uma metodologia engessada, podendo se apropriar de boas práticas de outras áreas do conhecimento, de acordo com a necessidade e o contexto de desenvolvimento de cada projeto. Essa revisão sistemática compõe o aporte teórico de uma pesquisa mais ampla e profunda que trata de uma proposta de design para jogos digitais endereçados a aprendizagem com base no GDD.

\section{REFERÊNCIAS BIBLIOGRÁFICAS}

BECKER, A. S.; CUMMINS, M.; DAVIS, A.; ESTRADA, V.; HALL, C. NMC Technology Outlook for International Schools in Asia: A Horizon Project Regional Report. Austin, Texas: The New Media Consortium, 2016. Disponível em: < http://cdn.nmc.org/media/2016-nmc-technology-outlook-isa.pdf >. Acesso em: 02 set. 2016.

CONTRERAS-ESPINOSA, R. S.; EGUIA-GÓMEZ, J. L. Investigación-acción como metodología para el diseño de un serious game. Revista Iberoamericana de Educación a Distancia, v.19, n.2, p 71-90, 2016. Disponível em: $<$ http://revistas.uned.es/index.php/ried/article/view/15624>. Acesso em: 28 ago. 2016.

COSTA, A. B.; ZOLTOWSKI, A. P. C. Como escrever um artigo de revisão sistemática. In: KOLLER, S. H., COUTO, M. C. P. P., HOHENDORFF, J. V. (orgs.). Manual de produção científica. Porto Alegre: Penso, 2014. 
DEBABI, W.; BENSEBAA, T. Using serious game to enhance algorithmic learning and teaching. Journal of e-Learning and Knowledge Society, v.12, n.2, p 127-140, mai. 2016. Disponível em: < http://www.je-lks.org/ojs/index.php/JeLKS_EN/article/view/1125>. Acesso em: 28 ago. 2016.

FREEMAN, A.; BECKER, A. S.; HALL, C. NMC Technology Outlook for Brazilian Universities: A Horizon Project Regional Report. Austin, Texas: New Media Consortium, 2015. Disponível em: < http://ppgtic.ufsc.br/files/2015/11/2015-nmctechnology-outlook-brazilian-universities-PT.pdf>. Acesso em: 03 set. 2016.

JOHNSON, L.; BECKER, A. S.; CUMMINS, M.; ESTRADA, V.; MEIRA, A. Technology Outlook for Brazilian Primary and Secondary Education 2012-2017: An NMC Horizon Project Regional Analysis. Austin, Texas: The New Media Consortium, 2012. Disponível em: <http://www.nmc.org/pdf/2012-technology-outlookbrazilian-primary-and-secondary-education.pdf $>$. Acesso em: 03 set. 2016.

JOHNSON, L.; BECKER, A. S.; ESTRADA, V.; FREEMAN, A.; KAMPYLIS, P.; PUNIE, Y. Horizon Report Europe: Schools Edition. Luxembourg: Publications Office of the European Union, \& Austin, Texas: The New Media Consortium, 2014. Disponível em: < https://ec.europa.eu/jrc/sites/jrcsh/files/horizon_report_eu_pt.pdf $>$. Acesso em: Acesso em: 02 set. 2016.

KATMADA, A.; MAVRIDIS, A.; TSIATSOS, T. Implementing a game for supporting learning in mathematics. The Electronic Journal of e-Learning, v.12, n.3, p 230-242, 2014. Disponível em: < www.ejel.org/issue/download.html?idArticle $=284>$. Acesso em: 28 ago. 2016.

MELERO, J.; HERNÁNDES-LEO, D. A Model for the Design of Puzzle-based Games Including Virtual and Physical Objects. Educational Technology \& Society, v.17, n.3, p 192-207, 2014. Disponível em: <http://www.ifets.info/journals/17_3/15.pdf > Acesso em: 28 ago. 2016.

MORANDI, M. I. W. M.; CAMARGO, L. F. R. Revisão sistemática da literatura. In: DRESCH, A.; LACERDA, D. P.; ANTUNES JÚNIOR, J. A. V. Design Science Research: método de pesquisa para avanço da ciência e tecnologia. Porto Alegre: Bookman, 2015.

NOVAK, Jeannie. Game Development Essentials. USA: Delmar Cangage Learning, 2012.

OZKUL, A. Development of a computer simulation game using a reverse engineering approach. American Journal of Business Education, v.5, n.6, nov. dez. 2012. Disponível em: < http://www.cluteinstitute.com/ojs/index.php/AJBE/article/view/7387 >. Acesso em: 28 ago. 2016.

SAHRIR, M. S.; ALIAS, N. A.; ISMAIL, Z.; OSMAN, N. Employing design and development research (DDR) approaches in the design and development of online Arabic vocabulary learning games prototype. The Turkish Online of Educational 
Technology, v.11, n.2, p 108-119, abr. 2012. Disponível em: < http://www.tojet.net/articles/v11i2/11211.pdf >. Acesso em: 28 ago. 2016.

STARKS, K. Cognitive behavioral game design: a unified model for designing serious games. Frontiers in Psychology, v.5, n.5, p 1-10, fev. 2014. Disponível em: < https://www.ncbi.nlm.nih.gov/pmc/articles/PMC3910127/>. Acesso em: 28 ago. 2016.

TANG, S.; HANNEGHAN, M.; CARTER, C. A platform independent game technology model for model driven serious games development. The Electronic Journal of e-Learning v.11, n.1, p 61-79, 2013. Disponível em: < www.ejel.org/issue/download.html?idArticle=228>. Acesso em: 28 ago. 2016 .

TORRENTE, J.; BORRO-ESCRIBANO, B.; FREIRE, M.; BLANCO, A. D.; MARCHIONI, I. M. O.; MORENO-GER, P.; FERNÁNDEZ-MANJÓN, B. Development of game-like simulations for procedural knowledge in healthcare education. IEEE Transactions on Learning Technologies, v.7, n.1, p 69-82, jan. mar. 2014. Disponível em: < http://ieeexplore.ieee.org/document/6678341/ >. Acesso em: 28 ago. 2016.

YANG, J. C.; CHIEN, K. H.; LIU, T. C. A digital game-based learning system for energy education: an energy conservation pet. The Turkish Online of Educational Technology, v.11, n.2, p 27-37, abr. 2012. Disponível em: < http://www.tojet.net/articles/v11i2/1123.pdf >. Acesso em: 28 ago. 2016. 